\title{
Sensitization Patterns in Immunoglobulin E-mediated Allergic Diseases by Skin Prick Testing Using Standardized Allergens: A Study from Tertiary Care Allergy Center
}

\author{
Nagaraju Kuravi ${ }^{1}$, Porchelvan Swaminathan ${ }^{2}$, Karthik Nagaraju ${ }^{3}$, Nihar R Mishra ${ }^{4}$, Bijan K Nayak ${ }^{5}$
}

\begin{abstract}
Aim and objective: To assess the sensitization patterns using standardized allergens in immunoglobulin E (IgE)-mediated allergic diseases by skin prick testing.

Materials and methods: This observational study was conducted in Chennai over a period of 1 year. Six hundred and three patients aged $\geq 6$ months of either gender with predefined inclusion and exclusion criteria were enrolled after taking written informed consent. Analysis of sensitization patterns with different standardized allergen extracts and their association with age groups were performed with SPSS $v 25.0$ (IBM, New York, USA).

Results: Out of 603 cases, male were predominant (M:F=1.8:1), with mean age of study population was 16.5 (13.2) years. There was no significant difference in polysensitization rates between under and above 18 years of age $(p>0.05)$. Out of 50 standardized allergens tested, the 5 most commonly sensitized were Blomia tropicalis (66.4\%), D. pteronyssinus (63.6\%), D. farinae (63.0\%), American cockroach (54.1\%), and Acarus siro $(48.8 \%)$. There was a statistically significant difference observed in the odds of sensitization between $<18$ years and $>18$ years of age groups for the following antigens - grass pollens, weed pollens, tree pollens, molds, animal epithelia, and insects $(p<0.05)$. However, no such association was found among mites and food $(p>0.05)$.

Conclusion: Majority of patients were polysensitized, with a high sensitization rate to house dust mite (HDM), predominantly B. tropicalis. There was also a higher rate of sensitizations to grass pollens than previous studies.

Clinical significance: Our study shows higher sensitization rates to HDMs, especially B. tropicalis, storage mites, and grass pollens. Standardized allergen extract use might improve the accuracy of sensitization patterns in the community.

Keywords: Age groups, IgE-mediated allergy, Polysensitization, Sensitization, Skin prick test, Standardized allergens, Type I allergies.

Clinical Rhinology An International Journal (2019): 10.5005/jp-journals-10013-1359
\end{abstract}

\section{INTRODUCTION}

The burden of allergic diseases in India has been increasing both in terms of prevalence and severity. ${ }^{1}$ It is estimated that $>25 \%$ of the Indian population suffer from various forms of allergies. ${ }^{2}$ Asthma, allergic rhinoconjunctivitis, atopic dermatitis, urticaria, and food allergies are common allergic disorders in Indian patients ${ }^{1}$ and a major contributor to the healthcare burden in the country. The allergen repertoire of Indian subcontinent is highly diverse ${ }^{3}$ due to the varied climate, flora, and food habits. The main source of allergens in Indian subcontinent are the dust mites, pollen grains, fungal spores, food, and insects. ${ }^{2,4}$ There is a strong relationship between these bio-particulate matters in environment and their effect on human health. ${ }^{3-5}$

Aeroallergen exposure is implicated as a strong risk factor for sensitization, development, and severity of immunoglobulin $E$ (IgE)-mediated atopic diseases. ${ }^{2,6}$ The principal role played by $\lg \mathrm{E}$ in type I hypersensitivity reactions is well recognized ${ }^{7,8}$ Skin prick test (SPT) is the most common and reliable method to diagnose IgE-mediated allergic diseases. ${ }^{9,10}$ It has advantages of relative sensitivity and specificity, fast results, flexibility, low cost, and good tolerability; this test also helps in the detection of offending allergens, thus helping in shaping right therapeutic interventions. ${ }^{9}$ To assess the clinical relevance of a positive SPT, it is important to understand the different factors that can influence the results of skin prick testing. ${ }^{11}$ Quality of composition and content of allergens in prick test solutions are mandatory in order to obtain reliable
${ }^{1}$ Department of Allergy and Clinical Immunology, Saveetha Medical College and Hospital, Chennai, Tamil Nadu, India

${ }^{2}$ Department of Biostatistics, Saveetha Medical College and Hospital, Chennai, Tamil Nadu, India

${ }^{3}$ VN Allergy and Asthma Research Centre, Chennai, Tamil Nadu, India

${ }^{4,5}$ Department of Pediatrics, Veer Surendra Sai Institute of Medical Sciences and Research, Sambalpur, Odisha, India

Corresponding Author: Nagaraju Kuravi, Department of Allergy and Clinical Immunology, Saveetha Medical College and Hospital, Chennai, Tamil Nadu, India, Phone: +91 9840230199, e-mail: majorknr@yahoo. co.in

How to cite this article: Kuravi N, Swaminathan P, Nagaraju K, et al. Sensitization Patterns in Immunoglobulin E-mediated Allergic Diseases by Skin Prick Testing Using Standardized Allergens: A Study from Tertiary Care Allergy Center. Clin Rhinol An Int J 2019;12(1):6-15.

Source of support: Nil

Conflict of interest: None

results. ${ }^{11}$ So, our aim is to assess the sensitization patterns using standardized allergens in SPT among patients visiting a tertiary care allergy research center in Chennai, Tamil Nadu, India.

\section{Materials and Methods}

This present analytical cross-sectional study was conducted in outpatient department of VN Allergy and Asthma Research Centre (Georeferenced; Fig. 1) Chennai, between May 2018 and April 2019

o The Author(s). 2019 Open Access This article is distributed under the terms of the Creative Commons Attribution 4.0 International License (https://creativecommons. org/licenses/by-nc/4.0/), which permits unrestricted use, distribution, and non-commercial reproduction in any medium, provided you give appropriate credit to the original author(s) and the source, provide a link to the Creative Commons license, and indicate if changes were made. The Creative Commons Public Domain Dedication waiver (http://creativecommons.org/publicdomain/zero/1.0/) applies to the data made available in this article, unless otherwise stated. 


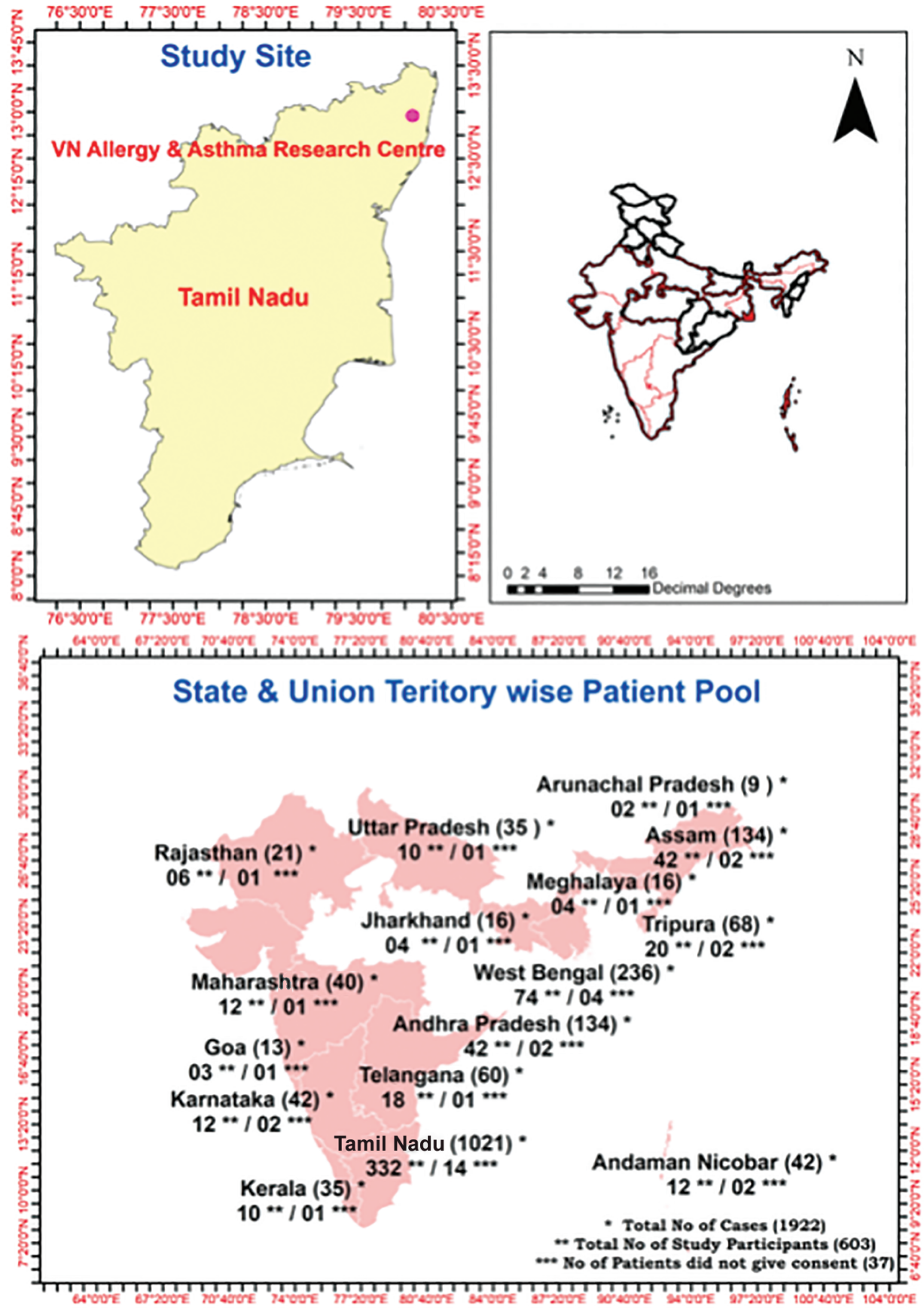

Fig. 1: Georeferenced areas of study subjects

after obtaining ethics approval (008/05/2018/IEC/SMCH). Patients with age $\geq 6$ months of either gender with signs and symptoms of allergy rhinitis ${ }^{12}$ or atopic dermatitis ${ }^{13}$ or asthma ${ }^{14}$ or food allergy ${ }^{15}$ or urticaria ${ }^{16}$ or anaphylaxis ${ }^{17}$ or angioedema ${ }^{18}$ were included in the study. Those having dermographism, ${ }^{19}$ acute asthma, ${ }^{14}$ and any other comorbidity like chronic lung diseases, congenital anomalies, pregnancy and lactation, and malignancy were excluded from the study.

Sample size estimation was performed by $\mathrm{n}$ master version 2.0 (BRTC, Vellore) by confidence interval (CI) estimating single proportion-absolute precision-finite population correction factor method. Population size was taken as $4,646,732,{ }^{20}$ hypothesized prevalence of sensitivity of allergens by SPT was $59 \%,{ }^{21}$ taking absolute precision of $5 \%$, confidence level of $95 \%$ and design effect of 1.5 , minimum sample was calculated to be $558\{n=[D E F F \times N p(1-p)] /[(d 2 / Z 21-a / 2 \times(N-1)+p \times(1-$ p)]\}. Out of 2,563 patients who visited our center during the study period, 1,922 cases were included from 16 different States and/or Union Territories (UTs) (Fig. 1) as per the predefined inclusion and exclusion criteria. From this study pool, 640 cases were selected by the two-stage sampling technique. First of all, each 16 States and/or UTs were considered as individual clusters and then samples from each cluster were taken by systematic random sampling method with sampling interval of 2 (Flowchart 1). 
Flowchart 1: Ninety-five study flowchart

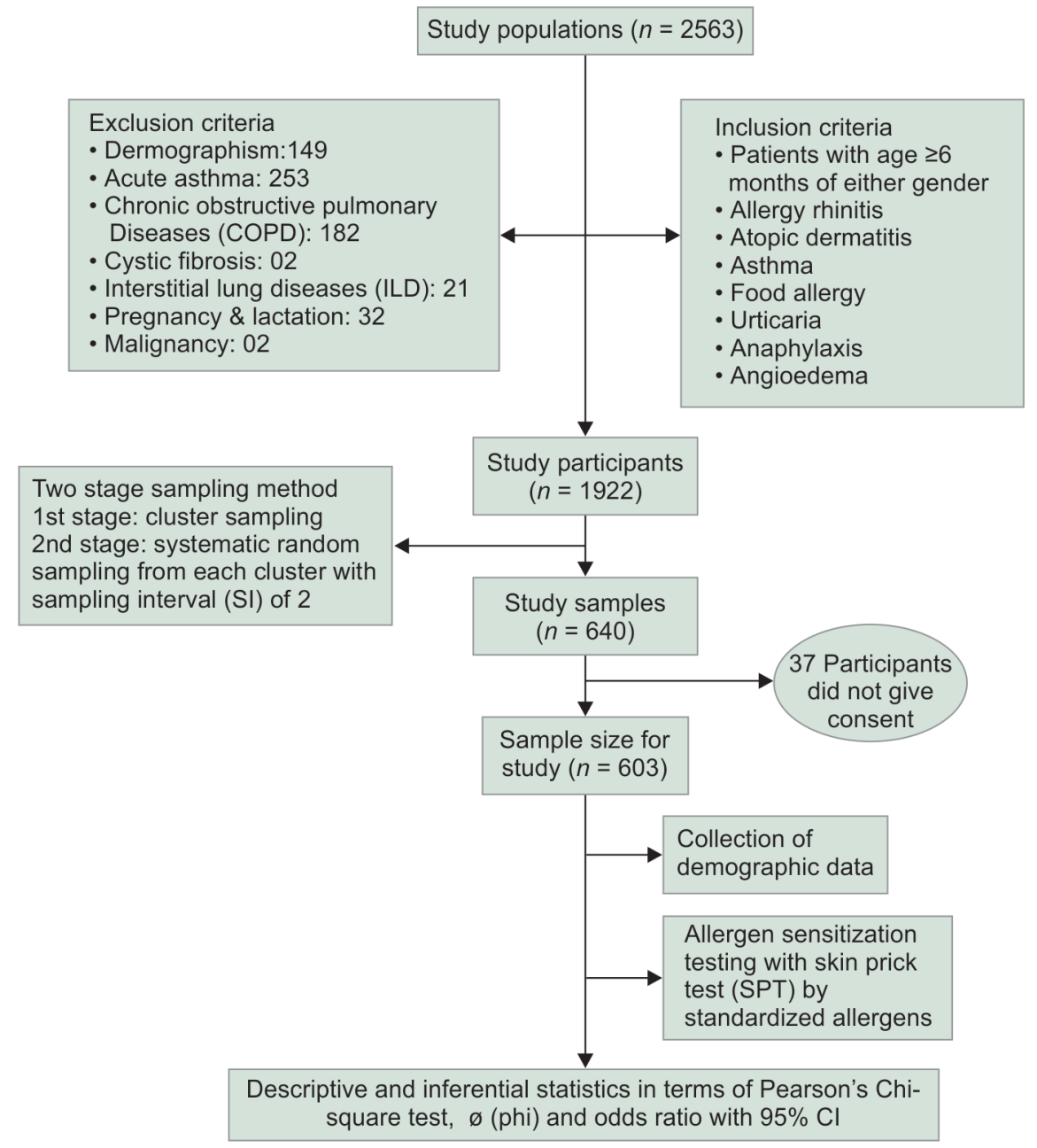

Standardized allergen extracts kit, AllergoSPT TM (Merck, Allergopharma) having $3 \mathrm{~mL}$ (color-coded) vial with dropper pipette containing the allergen dissolved in physiological saline solution with $50 \%$ glycerol and preserved with phenol were used. Skin prick test was performed using 50 different types of allergens, which included pollens, molds, house dust mite (HDM), food, animals, and insect. Standardized allergen extracts for German Cockroach and Blomia tropicalis (Inmunotek, Spain), American Cockroach and Mosquito (Greer laboratories, USA) were obtained separately. The number of allergens tested per patient was depending upon their history and presenting symptoms. Positive and negative controls used were histamine hydrochloride $1 \mathrm{mg} / \mathrm{mL}$ and $50 \%$ glycerinated saline, respectively. Skin prick tests were performed as per recommendations summarized in the position paper authored by Bosquet et al. ${ }^{10}$ Briefly, the procedure was applying a drop of allergen on the healthy skin on the flexor aspect of the forearm and then a prick was made using a sterile micro-lancet (Merck, Allergopharma). Skin prick test reaction reading was interpreted after 15 minutes. Skin reactivity assessment was performed by calculating the mean wheal diameter. Skin prick test was considered positive if mean wheal diameter was $3 \mathrm{~mm}$ compared with control. Oral drugs including antihistamines and any other drugs considered to influence outcomes of SPT were stopped 1 week before performing the tests. ${ }^{9}$
All the relevant data were recorded in a predesigned case report format (CRF). Data validation was performed manually by two separate persons not involved in the study. Continuous data were expressed in mean (SD); categorical data were expressed in proportions. Data normalcy testing of continuous data was performed by Shapiro-Wilk test and no transformation was required. Georeferencing was performed by Arc GIS v 9.3 (ESRI, California, USA). All the relevant statistics were performed by SPSS v 25.0 (IBM, New York, USA). Pearson's Chi-square test was applied to calculate the strength of association in terms of $\varnothing$ (phi). Odds ratio (OR) with $95 \% \mathrm{Cl}$ was calculated between age groups to know the strength of sensitization among various allergens. For all statistical purposes, $p$ value $<0.05$ was considered significant.

\section{Results}

Out of total 603 study participants, male outnumbered the female (M:F = 1.8:1), with mean (SD) age of study population was 16.5 (13.2) years. The study data comprised of patients from 16 different States and/or UTs of India (Fig. 1) with the majority (414) of patients from 5 southern states. The baseline demographic and clinical characteristics of study population are presented in Table 1. There was no statistically significant difference in percentage of polysensitization ( $>2$ sensitizations) between participants of age 
Table 1: Demographic characters of study population

\begin{tabular}{|c|c|c|c|c|}
\hline \multicolumn{5}{|c|}{ Study populations $(N=603)$} \\
\hline Variables & $n(\%)$ & Minimum & Maximum & $\operatorname{Mean}(S D)^{*}$ \\
\hline \multicolumn{5}{|l|}{ Gender } \\
\hline Male & $372(61.7)$ & & & \\
\hline Female & $231(38.3)$ & & & \\
\hline \multicolumn{5}{|l|}{ Age (years) } \\
\hline (1) $0.5-12$ & $333(55.2)$ & 0.6 & 12.0 & $7.7(2.8)$ \\
\hline (2) $12-18$ & 95 (15.75) & 12.5 & 18.0 & $14.7(1.5)$ \\
\hline (3) $18-30$ & $74(12.27)$ & 19.0 & 30.0 & $24.9(3.6)$ \\
\hline (4) $30-40$ & $60(9.95)$ & 31.0 & 40.0 & $35.1(2.7)$ \\
\hline (5) $40-65$ & $41(6.79)$ & 41.0 & 65.0 & $50.3(6.8)$ \\
\hline History of atopy & $598(99.2)$ & & & \\
\hline \multicolumn{5}{|l|}{ Sensitization } \\
\hline (1) One allergen & $23(3.8)$ & & & \\
\hline (2) Two allergens & $36(6)$ & & & \\
\hline (3) More than two allergens & $539(89.4)$ & & & \\
\hline (4) No & $05(0.8)$ & & & \\
\hline \multicolumn{5}{|l|}{ Disease condition } \\
\hline (1) $A R^{\#}$ with asthma & $292(48.42)$ & & & \\
\hline (2) AR & $122(20.23)$ & & & \\
\hline (3) Asthma & $30(4.97)$ & & & \\
\hline (4) AR with urticaria & $16(2.65)$ & & & \\
\hline (5) Others ${ }^{@}$ & $143(23.71)$ & & & \\
\hline \multicolumn{5}{|l|}{ Symptoms } \\
\hline (1) Perennial & $76(12.6)$ & & & \\
\hline (2) Seasonal & $112(18.57)$ & & & \\
\hline (3) Perennial with seasonal exacerbations & $415(68.82)$ & & & \\
\hline \multicolumn{5}{|l|}{ Symptoms severity } \\
\hline (1) Mild & $162(26.86)$ & & & \\
\hline (2) Moderate & $393(65.17)$ & & & \\
\hline (3) Severe & $48(7.96)$ & & & \\
\hline \multicolumn{5}{|l|}{ Symptom trigger } \\
\hline (1) Indoor & $247(41)$ & & & \\
\hline (2) Outdoor & $64(10.6)$ & & & \\
\hline (3) Both & $292(48.4)$ & & & \\
\hline
\end{tabular}

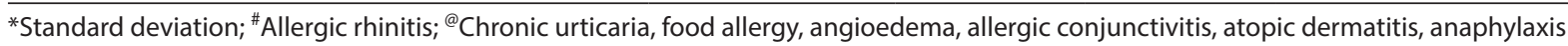

$<18$ years (96.8) as compared with those of $\geq 18$ years (95.7) of age groups (Chi-square value $=0.683, p>0.05 ; \varnothing: 0.112$ ). Most of the patients presented with allergic rhinitis with asthma (292). Among allergic rhinitis patients, most of them (415) presented as perennial with seasonal exacerbation.

The age-wise sensitization patterns to standardized allergens for mites (Table 2), molds (Table 3), grass pollens (Table 4), weed pollens (Table 5), tree pollens (Table 6), animals and insects (Table 7), and foods (Table 8) are described in detail. Out of 50 standardized allergens tested for sensitivity, most commonly reported positive SPT reactions in decreasing order (in percentage) were with $B$. tropicalis (66.4), D. pteronyssinus (63.6), D. farinae (63.0), American Cockroach (54.1), Acarus siro (48.8), Mosquito (42.3), Lepidoglyphus destructor (42.1), Tyrophagus putrescentiae (39.4), German Cockroach (36.8), and Shrimp (28.3).

Association of sensitization to molds, grass pollens, weed pollens, and tree pollens was statistically significant between less than and more than 18 years of age (OR: $0.74,95 \% \mathrm{Cl}: 0.61-0.88, p<$ 0.001; OR: $0.53,95 \%$ Cl: $0.44-0.63, p<0.001$; OR: $0.78,95 \%$ Cl: $0.65-$ $0.95, p<0.01$; OR: $0.69,95 \% \mathrm{Cl}: 0.56-0.87, p<0.001$, respectively). Aspergillus fumigatus was the most common mold (137) detected followed by Alternaria alternata (109). Kentucky Blue (115), Bermuda (137), and Timothy (103) grasses were the three most common grass allergens; while among tree pollens, Mosquito (99), Eucalyptus sp. (53), and Carica papaya (46) gained the majority in decreasing order. Parthenium hysterophorus (107), lambs quarter (125), and Amaranthus spinosus (87) were the most common weed allergens.

There was no significant association of sensitization to mites and food between age groups ( $<18$ and $\geq 18$ years) (OR: 1.14, 95\% Cl: 0.98 to $1.32, p>0.05$; OR: $0.92,95 \% \mathrm{Cl}: 0.82$ to $1.04, p>0.05$, respectively). Animal epithelia and insect allergen sensitization was found statistically significant between the two age groups of participants as mentioned above (OR: 2.28, 95\% Cl: 1.09-4.73, $p<$ 0.05; OR: 1.77; 95\% Cl: 1.07-2.91, $p<0.05$, respectively). American 
Table 2: Age-wise distribution of sensitization of mite allergens

\begin{tabular}{|c|c|c|c|c|c|c|}
\hline \multicolumn{7}{|c|}{ Mites $(N=603)$} \\
\hline $\operatorname{Mites}(n)^{*}$ & Overall, $n(\%)$ & $0.5-12$ years, $n(\%)$ & $12-18$ years, $n(\%)$ & $18-30$ years, $n(\%)$ & $30-40$ years, $n(\%)$ & $40-65$ years, $n(\%)$ \\
\hline D. farinae (603) & $380(63)$ & 209 (55) & $65(17.1)$ & $46(12.1)$ & $35(9.2)$ & $25(6.5)$ \\
\hline $\begin{array}{l}\text { D. pteronyssinus } \\
(602)\end{array}$ & $383(63.6)$ & $224(58.4)$ & $59(15.4)$ & $41(10.7)$ & $31(8.1)$ & $28(7.3)$ \\
\hline $\begin{array}{l}\text { Blomia tropicalis } \\
(366)\end{array}$ & $243(66.4)$ & $137(56.4)$ & $52(21.4)$ & $23(9.5)$ & $21(8.6)$ & $10(4.1)$ \\
\hline $\begin{array}{l}\text { Lepidoglyphus } \\
\text { destructor (354) }\end{array}$ & $149(42.1)$ & $80(53.7)$ & $25(16.8)$ & $24(16.1)$ & $09(6)$ & $11(7.4)$ \\
\hline Acarus siro (361) & $176(48.8)$ & $98(55.7)$ & $28(16)$ & $22(12.5)$ & $14(7.9)$ & $14(7.9)$ \\
\hline $\begin{array}{l}\text { Tyrophagus } \\
\text { putrescentiae (340) }\end{array}$ & $134(39.4)$ & $66(49.2)$ & $21(15.7)$ & $25(18.6)$ & $09(6.7)$ & $13(9.7)$ \\
\hline
\end{tabular}

*Each patient has been tested for multiple allergens depending upon his/her history and symptoms

Table 3: Age-wise distribution of sensitization of mold antigens

\begin{tabular}{|c|c|c|c|c|c|c|}
\hline \multicolumn{7}{|c|}{ Mold $(N=603)$} \\
\hline $\operatorname{Mold}(n)^{*}$ & Overall, n(\%) & $0.5-12$ years, $n(\%)$ & $12-18$ years, $n(\%)$ & $18-30$ years, $n(\%)$ & $30-40$ years, $n(\%)$ & 40-65 years, $n(\%)$ \\
\hline $\begin{array}{l}\text { Aspergillus } \\
\text { fumigatus (597) }\end{array}$ & $137(22.9)$ & $81(59.1)$ & $17(12.4)$ & $14(10.2)$ & $14(10.2)$ & $11(8.1)$ \\
\hline $\begin{array}{l}\text { Alternaria } \\
\text { alternata (596) }\end{array}$ & $109(18.3)$ & $58(53.1)$ & $16(14.7)$ & $12(11)$ & $10(9.2)$ & $13(12)$ \\
\hline $\begin{array}{l}\text { Botrytis cinerea } \\
(297)\end{array}$ & $25(8.4)$ & $13(52)$ & $04(16)$ & $03(12)$ & $04(16)$ & $01(4)$ \\
\hline $\begin{array}{l}\text { Cladosporium } \\
\text { herbarum (574) }\end{array}$ & $93(16.2)$ & 49 (52.7) & $13(14)$ & $13(14)$ & $08(8.6)$ & $10(10.7)$ \\
\hline $\begin{array}{l}\text { Fusarium } \\
\text { moniliforme (276) }\end{array}$ & $35(12.4)$ & $16(45.7)$ & $07(20)$ & $04(11.4)$ & $05(14.3)$ & $03(8.6)$ \\
\hline $\begin{array}{l}\text { Mucor mucedo } \\
\text { (16) }\end{array}$ & 0 & 0 & 0 & 0 & 0 & 0 \\
\hline $\begin{array}{l}\text { Penicillium } \\
\text { notatum (531) }\end{array}$ & $75(14.1)$ & $39(52)$ & $07(9.3)$ & $11(14.7)$ & $07(9.3)$ & $11(14.7)$ \\
\hline $\begin{array}{l}\text { Rhizopus nigricans } \\
\text { (357) }\end{array}$ & $56(15.7)$ & $23(41.1)$ & $09(16)$ & $12(21.4)$ & $08(14.3)$ & $04(7.2)$ \\
\hline $\begin{array}{l}\text { Helminthosporium } \\
\text { halodes (271) }\end{array}$ & $23(8.5)$ & $5(21.7)$ & $03(13)$ & $06(26.1)$ & $04(17.5)$ & $05(21.7)$ \\
\hline
\end{tabular}

*Each patient has been tested for multiple allergens depending upon his/her history and symptoms

Table 4: Age-wise distribution of sensitization of grass pollens

\begin{tabular}{|c|c|c|c|c|c|c|}
\hline \multicolumn{7}{|c|}{ Grass pollen $(N=603)$} \\
\hline Grass pollen $(n)^{*}$ & Overall, $n(\%)$ & $0.5-12$ years, $n(\%)$ & $12-18$ years, $n(\%)$ & $18-30$ years, $n(\%)$ & $30-40$ years, $n(\%)$ & $40-65$ years, $n(\%)$ \\
\hline $\begin{array}{l}\text { Bermuda grass } \\
\text { (592) }\end{array}$ & $137(23.1)$ & $74(54)$ & $12(8.8)$ & $21(15.3)$ & $16(11.7)$ & $14(10.2)$ \\
\hline $\begin{array}{l}\text { Timothy grass } \\
\text { (467) }\end{array}$ & $103(22.1)$ & $45(43.7)$ & $08(7.8)$ & $19(18.4)$ & $16(15.5)$ & $15(14.6)$ \\
\hline $\begin{array}{l}\text { Orchard grass } \\
(329)\end{array}$ & $49(14.9)$ & $24(49)$ & $05(10.2)$ & $11(22.4)$ & $07(14.3)$ & $02(4.1)$ \\
\hline $\begin{array}{l}\text { Corn zea mays } \\
(122)\end{array}$ & $20(16.4)$ & $12(60)$ & $01(5)$ & $03(15)$ & $01(5)$ & $03(15)$ \\
\hline Ryegrass (342) & $57(16.7)$ & $27(47.4)$ & $11(19.3)$ & 09 (15.8) & $03(5.2)$ & $07(12.3)$ \\
\hline $\begin{array}{l}\text { Kentucky } \\
\text { bluegrass (481) }\end{array}$ & $115(23.9)$ & $45(39.1)$ & $20(17.4)$ & $24(20.9)$ & $16(13.9)$ & $10(8.7)$ \\
\hline Barley (215) & $26(12.1)$ & $04(15.4)$ & $04(15.4)$ & 05 (19.2) & $09(34.6)$ & $04(15.4)$ \\
\hline $\begin{array}{l}\text { Cyperus rotundus } \\
\text { (362) }\end{array}$ & $68(18.7)$ & $31(45.6)$ & $14(20.6)$ & $11(16.2)$ & $08(11.8)$ & $04(5.9)$ \\
\hline
\end{tabular}

*Each patient has been tested for multiple allergens depending upon his/her history and symptoms 
Table 5: Age-wise distribution of sensitization of weed pollens

\begin{tabular}{|c|c|c|c|c|c|c|}
\hline \multicolumn{7}{|c|}{ Weed pollen $(N=603)$} \\
\hline Weed pollen $(n)^{*}$ & Overall, $n(\%)$ & $0.5-12$ years, $n(\%)$ & $12-18$ years, $n(\%)$ & $18-30$ years, $n(\%)$ & $30-40$ years, $n(\%)$ & 40-65 years, $n(\%)$ \\
\hline $\begin{array}{l}\text { Amaranthus spinosus } \\
\text { (393) }\end{array}$ & $87(22.1)$ & $50(57.5)$ & $16(18.4)$ & $09(10.3)$ & $05(5.7)$ & $07(8)$ \\
\hline $\begin{array}{l}\text { Parthenium } \\
\text { hysterophorus (459) }\end{array}$ & $107(23.3)$ & $56(52.3)$ & $23(21.5)$ & $07(6.5)$ & $15(14)$ & $06(5.6)$ \\
\hline $\begin{array}{l}\text { Lambs quarter (548) } \\
\text { (Chenopodium album) }\end{array}$ & $125(22.8)$ & $61(48.8)$ & $25(20)$ & $16(12.8)$ & $13(10.4)$ & $10(8)$ \\
\hline Ragweed (464) & $78(16.8)$ & $42(53.8)$ & $06(7.7)$ & $10(12.8)$ & $14(17.9)$ & $06(7.7)$ \\
\hline Engl plantain (352) & $62(17.6)$ & $24(38.7)$ & $06(9.7)$ & $15(24.2)$ & $10(16.1)$ & $07(11.3)$ \\
\hline Meadow fescue (181) & $13(7.2)$ & $03(23.1)$ & $03(23.1)$ & $03(23.1)$ & $03(23.1)$ & $01(7.7)$ \\
\hline $\begin{array}{l}\text { Mugwort-Artemisia } \\
\text { vulgaris (465) }\end{array}$ & $81(17.4)$ & $42(51.8)$ & $11(13.6)$ & $14(17.3)$ & $10(12.3)$ & $04(4.9)$ \\
\hline
\end{tabular}

*Each patient has been tested for multiple allergens depending upon his/her history and symptoms

Table 6: Age-wise distribution of sensitization of tree pollens

\begin{tabular}{|c|c|c|c|c|c|c|}
\hline \multicolumn{7}{|c|}{ Tree pollens $(N=603)$} \\
\hline Tree pollens $(n)^{*}$ & Overall, $n(\%)$ & $0.5-12$ years, $n(\%)$ & $12-18$ years, $n(\%)$ & $18-30$ years, $n(\%)$ & $30-40$ years, $n(\%)$ & 40-65 years, $n(\%)$ \\
\hline $\begin{array}{l}\text { Casuarina } \\
\text { equisetifolia (359) }\end{array}$ & $45(12.5)$ & $14(31.1)$ & $13(28.9)$ & $08(17.8)$ & $04(8.9)$ & $06(13.3)$ \\
\hline Eucalyptus sp. (338) & $53(15.7)$ & $20(37.7)$ & $14(26.4)$ & $04(7.5)$ & $09(17)$ & $06(11.3)$ \\
\hline $\begin{array}{l}\text { Prosopis juliflora } \\
\text { (356) }\end{array}$ & $99(27.8)$ & $38(38.4)$ & $24(24.2)$ & $13(13.2)$ & $15(15.2)$ & $09(9)$ \\
\hline $\begin{array}{l}\text { Peltophorum } \\
\text { pterocarpum (344) }\end{array}$ & $44(12.8)$ & $19(43.2)$ & $06(13.6)$ & $08(18.2)$ & $08(18.2)$ & $03(6.8)$ \\
\hline Cocos nucifera (342) & $34(9.9)$ & $12(35.3)$ & $10(29.4)$ & $02(5.9)$ & $08(23.5)$ & $02(5.9)$ \\
\hline Carica papaya (317) & $46(14.5)$ & $14(30.4)$ & $16(34.8)$ & $03(6.5)$ & 09 (19.6) & $04(8.7)$ \\
\hline $\begin{array}{l}\text { Holoptelea } \\
\text { integrifolia (358) }\end{array}$ & $39(10.9)$ & $19(48.7)$ & $13(33.3)$ & $01(2.6)$ & $04(10.2)$ & $02(5.1)$ \\
\hline $\begin{array}{l}\text { Ricinus communis } \\
\text { (115) }\end{array}$ & $13(11.3)$ & $07(53.8)$ & 0 & $02(15.4)$ & $03(23.1)$ & $01(7.7)$ \\
\hline $\begin{array}{l}\text { Xanthium commune } \\
\text { (105) }\end{array}$ & $07(6.7)$ & $02(28.6)$ & $01(14.2)$ & $02(28.6)$ & $02(28.6)$ & 0 \\
\hline
\end{tabular}

*Each patient has been tested for multiple allergens depending upon his/her history and symptoms

cockroach was the most common (266) insect found to be sensitized in our study. Among the food allergens, the most prevalent skin sensitization reactions were with shrimp (139) followed by milk (94).

\section{Discussion}

Out of 603 study participants, each patient was found to be sensitized to multiple allergens by SPT depending upon their history and symptoms; however, the rate of polysensitization was almost same in both under and above 18 years of age, while there was $26 \%$ less odds of sensitization with molds among $<18$-year patients as compared to that of $>18$ years. Forty-seven percent less odds of sensitization with grass pollens under 18-year patients as compared to that of above 18 years. There was $22 \%$ less odds of sensitization with weed pollens among $<18$-year patients as compared to that of $>18$ years. There was $31 \%$ less odds of sensitization with tree pollens among patients of two age groups as mentioned above. Twentyeight percent higher odds of sensitization with animal dander among $<18$-year patients as compared to that of $>18$ years. There was $77 \%$ higher odds of sensitization with insects among patients under 18 years of age as compared to that of above. The odds of sensitization with mites and food were not found to have significant association between the two age groups ( $<18$ and $\geq 18$ years).

Data from different parts of India have shown a sharp increase in the prevalence of type I allergic diseases (IgE-mediated) in the last few decades. Two routes of allergens exposure, such as inhalation, ingestion, and contact, have been found to have similar frequency among Indian atopic population. ${ }^{22-24}$ Despite having such a serious health risk, accurate diagnosis and therapeutic intervention of allergy still remain a challenge in the country.

A wide variety of factors may influence the result of SPTs. ${ }^{11}$ (1) The quality of allergen extract is of main significance as there is wide variation in composition and allergen content between allergen extracts from different manufacturers. (2) Technique of SPT. (3) The site used for skin prick testing. (4) The time of day. (5) Age, sex, and race, and (6) Concomitant drug treatment. ${ }^{11}$ Allergen extracts for SPT are native allergens obtained by extraction from the relevant biological material, such as pollen, mites, animal epithelia, and molds. National and international guidelines also recommend the use of standardized allergens for SPT, since quality of the allergen extracts can impact the test results. ${ }^{9,10,25}$ However, it is apparent that majority of allergen extracts used for SPT in India 
Table 7: Age-wise distribution of sensitization of animal dander, latex, and insects

\begin{tabular}{|c|c|c|c|c|c|c|}
\hline \multicolumn{7}{|c|}{ Animal, rubber, and insects panel $(N=603)$} \\
\hline Panel $(n)^{*}$ & Overall, $n(\%)$ & $0.5-12$ years, $n(\%)$ & $12-18$ years, $n(\%)$ & $18-30$ years, $n(\%)$ & $30-40$ years, $n(\%)$ & $40-65$ years, $n(\%)$ \\
\hline \multicolumn{7}{|l|}{ Animal dander $(n)$} \\
\hline Dog epithelia (281) & $30(10.7)$ & $18(60)$ & $02(6.7)$ & 05 (16.7) & $04(13.3)$ & $01(3.3)$ \\
\hline Cat epithelia (302) & $30(9.9)$ & $18(60)$ & $03(10)$ & $04(13.3)$ & $01(3.4)$ & $04(13.3)$ \\
\hline \multicolumn{7}{|l|}{ Latex $(n)$} \\
\hline Latex (111) & $03(2.7)$ & $02(66.7)$ & 0 & 0 & $01(33.3)$ & 0 \\
\hline \multicolumn{7}{|l|}{ Insects $(n)$} \\
\hline $\begin{array}{l}\text { Cockroach } \\
\text { American (492) }\end{array}$ & $266(54.1)$ & $138(51.9)$ & $47(17.7)$ & $32(12)$ & $29(11)$ & $20(7.5)$ \\
\hline $\begin{array}{l}\text { Cockroach German } \\
\text { (171) }\end{array}$ & $63(36.8)$ & $37(58.7)$ & $10(15.9)$ & $08(12.7)$ & $05(7.9)$ & $03(4.8)$ \\
\hline Mosquito (475) & $201(42.3)$ & $113(56.2)$ & $40(20)$ & $17(8.4)$ & 21 (10.4) & $10(5)$ \\
\hline
\end{tabular}

*Each patient has been tested for multiple allergens depending upon his/her history and symptoms

Table 8: Age-wise distribution of sensitization of foods

\begin{tabular}{|c|c|c|c|c|c|c|}
\hline \multicolumn{7}{|c|}{ Food panel $(N=603)$} \\
\hline Food panel (n)* & Overall, $n$ (\%) & $0.5-12$ years, $n(\%)$ & $12-18$ years, $n(\%)$ & $18-30$ years, $n(\%)$ & $30-40$ years, $n(\%)$ & $40-65$ years, $n(\%)$ \\
\hline Apple (418) & $64(15.3)$ & $43(67.2)$ & $04(6.2)$ & $05(7.8)$ & $05(7.8)$ & $07(11)$ \\
\hline Banana (514) & $52(10.1)$ & $36(69.2)$ & $03(5.8)$ & $07(13.4)$ & $04(7.7)$ & $02(3.8)$ \\
\hline Grape (427) & $47(11)$ & $27(57.4)$ & $08(17)$ & $03(6.4)$ & 05 (10.6) & $04(8.5)$ \\
\hline Orange (479) & 57 (11.9) & $30(52.6)$ & $11(19.3)$ & $06(10.5)$ & $06(10.5)$ & $04(7)$ \\
\hline Rice (191) & $13(6.8)$ & $02(15.4)$ & 0 & $04(30.8)$ & $05(38.5)$ & $02(15.4)$ \\
\hline Wheats (555) & $85(15.3)$ & $43(50.6)$ & $16(18.8)$ & $10(11.8)$ & $06(7)$ & $10(11.8)$ \\
\hline Chana dal (416) & $66(15.9)$ & $25(37.9)$ & $14(21.2)$ & $11(16.6)$ & $13(19.7)$ & $03(4.5)$ \\
\hline Black gram (272) & 35 (12.9) & $14(40)$ & $08(22.8)$ & $05(14.3)$ & $03(8.6)$ & $05(14.3)$ \\
\hline Green gram (340) & $46(13.5)$ & $19(41.3)$ & $11(23.9)$ & $07(15.2)$ & $07(15.2)$ & $02(4.3)$ \\
\hline Toor dal (433) & $45(10.4)$ & $18(40)$ & $12(26.6)$ & $07(15.5)$ & $05(11.1)$ & $03(6.7)$ \\
\hline Soy bean (411) & $42(10.2)$ & $27(64.3)$ & $06(14.3)$ & 0 & 07 (16.7) & $02(4.8)$ \\
\hline Ground nut (549) & $56(10.2)$ & $37(66.1)$ & $03(5.4)$ & $06(10.7)$ & $07(12.5)$ & $03(5.4)$ \\
\hline Cashewnut (477) & $51(10.7)$ & $22(43.1)$ & $12(23.5)$ & $04(7.8)$ & $10(19.6)$ & $03(5.9)$ \\
\hline Almonds (511) & $68(13.3)$ & $34(50)$ & $11(16.2)$ & $07(10.3)$ & $05(7.3)$ & $11(16.2)$ \\
\hline Chocolate (560) & $40(8.7)$ & $24(60)$ & $08(20)$ & $01(2.5)$ & $06(15)$ & $01(2.5)$ \\
\hline Pista (439) & $58(13.2)$ & $34(58.6)$ & $10(17.2)$ & $02(3.4)$ & $08(13.8)$ & $04(6.9)$ \\
\hline Tomato (362) & $30(8.3)$ & $10(33.3)$ & $04(13.3)$ & $05(16.7)$ & $07(23.3)$ & $04(13.3)$ \\
\hline Brinjal (329) & $31(9.4)$ & $12(38.7)$ & 04 (12.9) & $05(16.1)$ & $06(19.3)$ & $04(12.9)$ \\
\hline Ladies finger (362) & $29(8.0)$ & $14(48.3)$ & 05 (17.2) & $02(6.9)$ & $03(10.3)$ & $05(17.2)$ \\
\hline Milk (583) & $94(16.1)$ & $63(67)$ & $07(7.4)$ & $09(9.6)$ & $09(9.6)$ & $06(6.4)$ \\
\hline Egg (566) & $86(15.2)$ & $52(60.5)$ & 10 (11.6) & $08(9.3)$ & $09(10.5)$ & $07(8.1)$ \\
\hline Cod (352) & 39 (11.1) & $24(61.5)$ & 04 (10.2) & 07 (17.9) & $02(5.1)$ & $02(5.1)$ \\
\hline Mussel (205) & $11(5.4)$ & $08(72.7)$ & 0 & $01(9.1)$ & $01(9.1)$ & $01(9.1)$ \\
\hline Carp (288) & $24(8.3)$ & $14(58.3)$ & $02(8.3)$ & $02(8.3)$ & $04(16.6)$ & $02(8.3)$ \\
\hline Shrimp (491) & $139(28.3)$ & $69(49.6)$ & $26(18.7)$ & $23(16.5)$ & $11(7.9)$ & $10(7.2)$ \\
\hline Chicken (466) & $50(11.2)$ & $26(52)$ & $12(24)$ & $06(12)$ & $02(4)$ & $04(8)$ \\
\hline Mutton (414) & $40(9.7)$ & $24(60)$ & $06(15)$ & 05 (12.5) & $03(7.5)$ & $02(5)$ \\
\hline Crab (319) & 37 (11.6) & $13(35.1)$ & $10(27)$ & $06(16.2)$ & $06(16.2)$ & $02(5.4)$ \\
\hline Gluten (264) & $40(15.2)$ & $20(50)$ & $10(25)$ & $02(5)$ & $06(15)$ & $02(5)$ \\
\hline Masoor dal (140) & $17(12.1)$ & 09 (52.9) & $08(47.1)$ & 0 & 0 & 0 \\
\hline
\end{tabular}

*Each patient has been tested for multiple allergens depending upon his/her history and symptoms 
are not standardized and no action plan has been established for improving the quality of these diagnostic reagents. ${ }^{2}$ Therefore, in our study, we used standardized allergen extracts.

This cross-sectional study is among the first studies to test for allergen sensitization using standardized allergens among Indian patients. In this study, 99.2\% patients were atopic and $95.4 \%$ were polysensitized. These results are similar to $100 \%$ atopy reported by Sharma et al., ${ }^{26}$ but comparatively higher than $71.57 \%$ reported by Kumar et al., 71.94 and $67 \%$ reported by Gowda et al. ${ }^{28}$ and Nagaraj and Chethna, ${ }^{29}$ respectively.

Climatic conditions, urbanization, and more indoor lifestyle are implied as probable cause of increased HDM exposure and sensitization. ${ }^{30}$ The results from our study also show that HDMs were most commonly sensitized aeroallergens. House dust mites particularly $D$. pteronyssinus and $D$. farinae have been shown to play an important role in the pathogenesis of asthma and allergic rhinitis, ${ }^{31-34}$ but from the Indian perspective, they are not well characterized at the molecular level. ${ }^{30}$ Our study identified that $B$. tropicalis was the most common dust mite. Blomia tropicalis was previously classified as storage mite, but recently classified as HDM. ${ }^{35}$ It has been found to be highly prevalent in tropical and subtropical climates, especially in South East Asian countries. ${ }^{35}$ This study presents for the first time a high prevalence of $B$. tropicalis among a large group of Indian patients represented from various parts of the country. This could possibly be due to availability of standardized allergen extracts. The present study also documented sensitization pattern of the storage mites $A$. siro, L. destructor, and $T$. putrescentiae among Indian patients. Another study from Eastern India found that the sensitization rates for $A$. siro and $L$. destructor were 33 and $25 \%$, respectively. ${ }^{36} \mathrm{~A}$ higher finding in our study could be due to patients from various parts of India.

Various studies have reported insects to be the most common offending antigens in India, with sensitization in the range of 17.5 to $43.9 \%$ of the antigens. ${ }^{27,37-39}$ In India, cockroach allergens have been considered as one of the triggering factors for the development of atopic asthma, with American cockroach ( $P$. americana) as most commonly found species. ${ }^{2}$ Data from our study have also shown high sensitization to American cockroach (54.1\%) which is comparatively higher than 3.5 and $25.7 \%$ reported by Gowda et al. $^{28}$ and Chogtu et al., ${ }^{39}$ respectively, in patients with respiratory allergies. The higher sensitizations reported in our study can be attributed to regional difference in patient population and the use of standardized allergens in our study. Another possible explanation for this could be cross-reactivity between HDM and cockroach allergens. Studies with component resolved diagnosis might help in further understanding.

Among pollen allergens, tree pollen Prosopis juliflora (12\%) was the most common allergen followed by grass pollen Poa pratensis (16.9\%) and weed pollen P. hysterophorus (34\%), which is corroborating with a previous study. ${ }^{40}$ In a recent aerobiology study from Delhi by Kumar et al. ${ }^{41}$ also reported grass pollens belonging to Poaceae family (6.83\%) to be the dominant pollens. In our study, we report the sensitization to $P$. pratensis, Phleum pretense, Lolium perenne, and Dactylis glomerata for the first time in patients of South India. A higher incidence of grass pollen allergy in south India could be due to rapid urbanization, artificial grass use, and also improved quality of allergen extracts. From Southern India, $P$. hysterophorus has been reported as an important source of aeroallergens. ${ }^{28,42,43}$ Recently, Gowda et al. ${ }^{28}$ reported $20.86 \%$ allergenicity to $P$. hysterophorus among pollens in patients with bronchial asthma and/or allergic rhinitis, which is similar to our study. Whether it is due to cosensitization or cross-reactivity or due to pan allergens need to established by component testing, which is not available in India. ${ }^{24}$

Among molds, A. fumigatus (22.9\%) was the most common allergen followed by A. alternata (18.3\%), which is comparatively higher than $4.3 \%{ }^{27}$ for $A$. fumigatus and $5.7 \%^{28}$ reported for $A$. alternata. This higher result in our study may be due to use of standardized allergens, as different species of Aspergillus are highly predominant in the ambient air of India, ${ }^{2}$ and due to reduced indoor ventilation.

Among the animal dander, dog (10.7\%) was the most common sensitizer, while cat allergens were positive in $9.9 \%$ of our patients, which is comparable with Chogtu et al. (9.8\% for dog dander). ${ }^{39}$ Prevalence of allergy to furry animals (dog and cats) has been increasing in the last decade probably due to increase practice of growing pets in India. Among the food allergens tested in our study, we observed a high sensitization to shrimps (28.3\%) in our patients, followed by milk (16.1\%), chana dal (15.9\%), and apple (15.3\%). India is a country with diverse food habits and cuisines, with consumption varying from vegetables to dairy products, etc. The most common types of food allergy prevailing among the Indian population include legume allergy, prawn allergy, milk allergy, and egg allergy. ${ }^{44}$ Milk allergy was most commonly seen between 5 and 15 years of age group, prawn allergy between 16 and 40 years of age group, and legume allergy was most common between 41 and 60 years of age group, ${ }^{45}$ but we have not found any age group discrimination on food allergy patterns above and under 18 years of age. The reason may be due to diversity of climatic condition, different inclusion and exclusion criteria, and nature of allergen extract was not clearly mentioned. There is presently no article on classification of age group on other allergen sensitization. The high shrimp sensitization in our study may be due to cross-reactivity with dust mites and cockroach species. Component testing may help in proper identification.

We have evaluated data of patients from 16 different states of India, visiting specialist allergy center. Another important aspect of our study was the use of standardized allergen extracts kits. We also identified sensitization to $B$. tropicalis, storage mites, and grass pollens (South India) mainly due to use of standardized allergens. The study was also relatively simple, less time-consuming, and with limited resources we were able to get baseline data which will help us in future for further studies. We believe it will enrich our existing knowledge on use of standardized allergens in SPT.

However, present study has certain limitations like crosssectional study design, so causality could not be established. Majority of patients belonged to Tamil Nadu, so the results could not be generalized. Confounders and effect modifiers could not be avoided due to study design per se. Region-wise allergen sensitization has not been studied. Further studies with larger cohort of allergy patients will help in better understanding of the sensitization patterns region-wise.

\section{Conclusion}

Accurate identification of the offending allergen can provide an opportunity for effective management of patients suffering from an allergic disorder. The results of this study show that majority of patients who underwent SPT using standardized allergens 
were polysensitized, with a high sensitization rate to HDM. The rate of polysensitization was comparable between pediatric and adult age groups. Blomia tropicalis should be considered as a significant indoor aeroallergen along with $D$. farinae and $D$. pteronyssinus in our country. We recommend the practitioners to include $B$. tropicalis, storage mites, and grass pollen in their allergy test kits. However, a holistic approach with larger sample size will give us a better level of understanding and plan future therapeutic strategies.

\section{Clinical Significance}

Our study shows higher sensitization rates to HDMs, especially $B$. tropicalis, storage mites, and grass pollens. Standardized allergen extract use might improve the accuracy of sensitization patterns in the community.

\section{References}

1. Prasad R, Kumar R. Allergy situation in India: what is being done? Indian J Chest Dis Allied Sci 2013;55:7-8.

2. Bhattacharya $K$, Sircar G, Dasgupta A, et al. Spectrum of allergens and allergen biology in India. Int Arch Allergy Immunol 2018;177(3):219237. DOI: $10.1159 / 000490805$.

3. Singh $A B$, Kumar P. Common environmental allergens causing respiratory allergy in India. Indian J Pediatr 2002;69(3):245-250. DOI: 10.1007/BF02734234.

4. Singh $A B$, Kumar P. Aeroallergens in clinical practice of allergy in India. An overview. Ann Agric Environ Med 2003;10:131-136.

5. Singh $A B$. Pollen and fungal aeroallergens associated with allergy and asthma in India. Global J Immunol Allerg Diseas 2014;2(1):19-28. DOI: 10.14205/2310-6980.2014.02.01.4.

6. Tham EH, Lee AJ, Bever HV. Aeroallergen sensitization and allergic disease phenotypes in Asia. Asian Pac J Allergy Immunol 2016;34:181189.

7. Vora A. Anti lgE therapy in allergic asthma and allergic rhinitis. Med Update 2012. 370-377. Available at URL: http://www.apiindia.org/ pdf/medicine_update_2012/pulmonology_02.pdf.

8. Gelfand EW. Anti-lgE therapy in asthma http://www.medscape.org/ viewarticle/530088.

9. Heinzerling L, Mari A, Bergmann KC, et al. The skin prick test European standards. Clin Transl Allergy 2013;3(1):3. DOI: 10.1186/20457022-3-3.

10. Bousquet J, Heinzerling $L$, Bachert $C$, et al. Practical guide to skin prick tests in allergy to aeroallergens. Allergy 2012;67(1):18-24. DOI: 10.1111/j.1398-9995.2011.02728.x.

11. Wagner N, Rubert M. Sensitivity and specificity of standardised allergen extracts in skin prick test for diagnoses of IgE-mediated respiratory allergies. Clin Transl Allergy 2019;9(1):8. DOI: 10.1186/ s13601-019-0248-9.

12. Wheatley LM, Togias A. Clinical practice. allergic rhinitis. N Engl J Med 2015;372(5):456-463. DOI: 10.1056/NEJMcp1412282.

13. Thomsen SF. Atopic dermatitis: natural history, diagnosis, and treatment. ISRN Allergy 2014;2014:354250. DOI: 10.1155/2014/354250.

14. Horak F, Doberer D, Eber E, et al. Diagnosis and management of asthma - statement on the 2015 GINA guidelines. Wien Klin Wochenschr 2016;128(15):541-554. DOI: 10.1007/s00508-0161019-4.

15. Sampson HA. Update on food allergy. J Allergy Clin Immunol 2004;113(5):805-819. DOI: 10.1016/j.jaci.2004.03.014.

16. Bernstein JA, Lang DM, Khan DA, et al. The diagnosis and management of acute and chronic urticaria: 2014 update. J Allergy Clin Immunol 2014;133(5):1270-1277. DOI: 10.1016/j.jaci.2014.02.036.

17. Ring J, Brockow K, Duda D, et al. Emergency treatment of anaphylactic reactions. Allergo J 2007;16(6):420-434. DOI: 10.1007/BF03370705.
18. Kaplan AP. Angioedema. World Allergy Organ J 2008;1(6):103-113. DOI: 10.1097/WOX.0b013e31817aecbe.

19. Richard K, Ebken BA, Frederick A, et al. Dermographism: Its definition, demonstration, and prevalence. J Aller Clin Immunol 1968;41(6):338343. DOI: 10.1016/0021-8707(68)90076-2.

20. Centre of India 2011. District Census Handbook, Chennai. Available at www.censusindia.gov.in/2011census/dchb/3302_PART_DCHB_ CHENNAI.

21. Mozhgan M, Saeed T, Shirin F. Sensitization to common allergens among patients with allergies in major Iranian cities: a systematic review and meta-analysis. Epidemiol Health 2017;39:e2017007. DOI: 10.4178/epih.e2017007.

22. Boral D, Chatterjee S, Bhattacharya K. The occurrence and allergizing potential of airborne pollen in West Bengal, India. Ann Agric Environ Med 2004;11(1):45-52.

23. Sharma VK, Chakrabarti A. Common contact sensitizers in Chandigarh. India A Study 200 Patien Eur Stand Ser Contact Dermat 1998;38:127-131.

24. Wong GWK, Mahesh PA, Ogorodova L, et al. The Euro prevall-INCO surveys on the prevalence of food allergies in children from China, India and Russia: the study methodology. Allergy 2010;65(3):385-390. DOI: 10.1111/j.1398-9995.2009.02214.x.

25. 2018 GINA Report, Global Strategy for Asthma Management and Prevention. [September 19, 2019]. Available From: https:// ginasthma.org/wp-content/uploads/2018/03/WMS-FINAL-GINA2018-Appendix_v1.3.pdf.

26. Sharma RK, Mathur Y, Chhabra G, et al. A study of skin sensitivity to various allergens by skin prick test in patients of bronchial asthma and allergic rhinitis. Indian J Allergy Asthma Immunol 2018;32(2):47-53. DOI: 10.4103/ijaai.ijaai_9_18.

27. Kumar R, Sharan N, Kumar M, et al. Pattern of skin sensitivity to various aeroallergens in patients of bronchial asthma and/or allergic rhinitis in India. Indian J Allergy, Asthma Immunol 2012;26(2):66-72. DOI: 10.4103/0972-6691.112550.

28. Gowda G, Nagaraj C, Parasuramalu BG, et al. Aeroallergen sensitivity among patients suffering from bronchial asthma in Bangalore. Int J Health Allied Sci 2013;2(4):237-241. DOI: 10.4103/2278-344X.126710.

29. Nagaraj C, Chethna G. Spectrum of aero allergen sensitivity among patients suffering from nasobronchial allergy, Bangalore, Karnataka. Int J Recent Scientific Res 2015;6:4900-4903.

30. Dey D, Saha GK, Poddar S. A review of house dust mite allergy in India. Exp Appl Acarol 2019;78(1):1-14. DOI: 10.1007/s10493-019-00366-4.

31. Gill NA, Singh A, Dhaliwal AK, et al. House dust mites and pollens as risk factors in allergic manifestations. Indian J Sci Res 2016;7(1): 131-142.

32. Fernandez-Caldas E, Puerta L, Caraballo L. Mites and allergy. Chem Immunol Allergy 2014;100:234-242.

33. Miller JD. The role of dust mites in allergy. Clin Rev Allergy Immunol 2018(3). DOI: 10.1007/s12016-018-8693-0.

34. Gaur SN. An Indian perspective on dust mites. Indian J Allergy Asthma Immunol 2019;33(1):14-18. DOI: 10.4103/ijaai.ijaai_9_19.

35. Guilleminault L, Viala-Gastan C. Blomia tropicalis: a house dust mite in the tropics. Rev Mal Respir (Abstract) 2017;34(8):791-801. DOI: 10.1016/j.rmr.2016.10.877.

36. Mondal P, Dey D, Sarkar T, et al. Evaluation of sensitivity toward storage mites and house dust mites among nasobronchial allergic patients of Kolkata, India. J Med Entomol 2019;56(2):347-352. DOI: 10.1093/jme/tjy206.

37. Patel A, Chaudhary S. Prevalence of allergen sensitivity in nasobronchial allergy in Gujarat, India Natl. J Med Res 2012;2:431-434.

38. Prasad R, Verma SK, Dua R, et al. A study of skin sensitivity to various allergens by skin prick test in patients of nasobronchial allergy. Lung India 2009;26(3):70-73. DOI: 10.4103/0970-2113.53228.

39. Chogtu B, Magaji N, Magazine R, et al. Pattern of allergen sensitivity among patients with bronchial asthma and/or allergic Rhinosinusitis in a tertiary care centre of Southern India. J Clin Diagn Res 2017;11:OC01-OC04. DOI: 10.7860/JCDR/2017/26973.10328. 
40. Singh $A B$, Kumar Pawan. Aerial pollen diversity in India and their clinical significance in allergic diseases. Indian J Clin Biochem 2004;19(2):190-201. DOI: 10.1007/BF02894284.

41. Kumar R, Kumar D, Singh K, et al. Identification of airborne pollens in Delhi. Indian J Allergy Asthma Immunol 2018;32(1):28-33. DOI: 10.4103/ijaai.ijaai_35_17.

42. Acharya PJ. Skin test response to some inhalant allergens in patients of naso-bronchial allergy from Andhra Pradesh. Asp Allergy Appl Immunol 1980;13:14-18.
43. Rao M, Prakash O, Subba Rao PV. Reaginic allergy to parthenium pollen: evaluation by skin test and RAST. Clin Allergy 1985;15(5):449454. DOI: 10.1111/j.1365-2222.1985.tb02294.x.

44. Arakali SR, Green TD, Dinakar C. Prevalence of food allergies in South Asia. Ann Allergy Asthma Immunol 2017;118(1):16-20. DOI: 10.1016/j. anai.2016.09.441.

45. Mandal J, Das M, Roy l, et al. Immediate hypersensitivity to common food allergens: an investigation on food sensitization in respiratory allergic patients in Calcutta. India World Allergy Organ J 2009;2(1):0912. DOI: $10.1097 /$ WOX.0b013e318194c0de. 